\title{
$A b$ initio spin-free-state-shifted spin-orbit configuration interaction calculations on singly ionized iridium
}

\author{
Frank Rakowitz \\ Institut für Physikalische und Theoretische Chemie, Universität Bonn, Wegelerstr. 12, 53115 Bonn, \\ Germany \\ Marcos Casarrubios and Luis Seijo ${ }^{a}$ \\ Departamento de Química, C-XIV, Universidad Autónoma de Madrid, 28049 Madrid, Spain \\ Christel M. Marian ${ }^{\text {b) }}$ \\ Institut für Physikalische und Theoretische Chemie, Universität Bonn, Wegelerstr. 12, 53115 Bonn, \\ Germany
}

(Received 24 November 1997; accepted 11 February 1998)

\begin{abstract}
This work presents a systematic test of the performance of a spin-orbit operator founded upon the Wood-Boring-based ab initio model potential method [J. Chem. Phys. 102, 8078 (1995)]. Assuming a separability of the problem into a spin-free correlation treatment and a spin-orbit calculation part, this aim can be reached. We shall show in this publication both the separability and the high level of quality of the spin-orbit operator applying our method to the even spectrum of $\mathrm{Ir}^{+}$. We shall treat the spin-orbit part by means of the above mentioned spin-orbit operator and cope with the spin-free correlation problem through introducing a spin-free-state-shifting operator, shifting the spin-free energies to empirical values obtained from experiment. The quality of the spin-orbit operator is very high, actually better than estimated in previous calculations which were contaminated by an insufficient treatment of correlation. The procedure established is most efficient: Spin-free-stateshifted spin-orbit CI calculations employing a space of the significant reference configurations plus single excitations lead to very reliable spin-orbit splittings provided that the spin-free states are calculated at a high level of quality. (C) 1998 American Institute of Physics.
\end{abstract}

[S0021-9606(98)01619-5]

\section{INTRODUCTION}

The relevance of relativistic effects to the chemistry of heavy elements on the one hand and the general importance of electron correlation effects on the other hand, has driven and still drives the search for suitable $a b$ initio computational methods in this concern. ${ }^{1}$ Today, a number of methods exist that do include spin-orbit coupling effects in correlated molecular calculations, ranging from sophisticated all-electron four-component configuration-interaction methods based on the Dirac-Hartree-Fock $(\mathrm{DHF}+\mathrm{CI})$ formalism ${ }^{2}$ to more pragmatic effective-core-potential (ECP) spin-orbit configuration-interaction (spin-orbit CI) methods. ${ }^{3-5}$ The latter group comprises the so-called Wood-Boring-based $a b$ initio model potential method (WB-AIMP), ${ }^{6}$ an elaboration of ideas of Katsuki and Huzinaga. ${ }^{7,8}$ A spin-dependent Hamiltonian with effective one-electron atomic spin-orbit operators based on the Dirac equation for the large components, ${ }^{9}$ as proposed by Wood and Boring, ${ }^{10}$ is employed. These operators implicitly include two-electron contributions since they are derived from the one-electron atomic mean-field potentials. A detailed knowledge of these

\footnotetext{
a) Author to whom correspondence should be addressed; Electronic mail: 1s@sara.qfa.uam.es

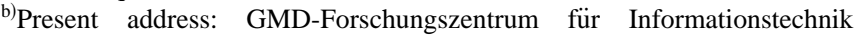
$\mathrm{GmbH}$, Institut für Algorithmen und Wissenschaftliches Rechnen (SCAI), Schloss Birlinghoven, 53754 Sankt Augustin, Germany.
}

contributions and of their size, though, does not exist; their potentiality has been discussed ${ }^{10-13}$ and their superiority over the explicit inclusion of two-electron contributions by means of the procedure of Blume and Watson ${ }^{14,15}$ has been pointed out. ${ }^{16}$ In practical calculations, these atomic one-electron spin-orbit operators have been modified to the form proposed by Pitzer and Winter, ${ }^{4}$ and they have been produced for the elements of the periodic table up to $\mathrm{Ba},{ }^{17}$ the third-row transiton metal elements, ${ }^{18,19}$ and $\mathrm{Tl}-\mathrm{Rn}^{6}{ }^{6}$ Their quality was monitored in spin-orbit CI calculations and found to be reasonable; ${ }^{6,18}$ one of us has proposed the use of an empirical scaling parameter as a means to correct (mainly) an insufficient treatment of the two-electron contributions. Obtained values of such a parameter appear to come out systematically close to unity. ${ }^{6,18}$ However, since both the amounts of correlation effects and spin-orbit couplings significantly influence the observed spin-orbit splittings, the previously mentioned monitoring calculations may be in part biased by an insufficient treatment of electron correlation and, in consequence, the empirical scaling factor might correct this insufficiency rather than the two-electron spin-orbit contributions. A realistic check for the quality of any spin-orbit operator, in particular of Wood and Boring's, can only be achieved with calculations that do not show any deficiency in the treatment of the spin-free effects.

In previous work, in an attempt to decouple correlation and spin-orbit effects, spin-orbit calculations were performed 
on the basis of perturbation theory using spin-free energies from higher level electron correlation treatments. ${ }^{3,20,21}$ In the line of this work a simple two-step method (spin-free-stateshifting technique, $s f s s$ ) was proposed ${ }^{22}$ which is specially indicated for spin-orbit CI calculations in a basis of determinants or of double-group symmetry adapted functions: ${ }^{4}$ The first step uses a spin-free Hamiltonian with a calculation that treats correlation effects to a high level of quality, e.g., a large CI. In the second step, the spin-orbit Hamiltonian is used in a spin-orbit CI calculation of smaller size being sufficient for the spin-orbit couplings but generally insufficient for the correlation effects and, accordingly, for the spin-free energies. The latter Hamiltonian is extended by adding an operator which shifts the spin-free states to the energies that had resulted from the high quality calculation of the first step. In this way electron correlation and spin-orbit effects are decoupled to a large extent, and two useful consequences show up: On the one hand, in the treatment of electron correlation only less severe limitations of spin-free calculations apply, on the other hand the evaluation of spin-orbit interaction in the second step is not as restrictive as a (quasidegenerate) perturbation treatment in just a few spin-free CI wave functions. This method is suitable for an alternative use: One can substitute the first step by a gathering of empirical spin-free energy data, if possible, and subsequently shift the spin-free states in the second step to their empirical values. According to the idea behind the spin-free-stateshifting technique, this would then correspond to a full representation of the correlation effects. In consequence, the use of the $s f s s$ technique with empirical shifts in spin-orbit CI calculations is appropriate for proper monitoring of a spinorbit operator, with a minimal danger of having the results contaminated by an insufficient treatment of correlation.

In this paper, we use an empirically spin-free-stateshifted Hamiltonian in the calculation of the even spectrum of $\mathrm{Ir}^{+}$in order to precisely check for the quality of the WBAIMP spin-orbit operator in applications to third row transition metals. Further the performance of the $s f s s$ technique is tested, and its conditions of use are explored. This work is a first step of theoretical studies on $\mathrm{Ir}^{+}$-mediated activation reactions; a detailed knowledge of the ability of the method to accurately describe the atomic spectrum of $\mathrm{Ir}^{+}$is relevant for estimating the reliability of molecular results.

\section{METHOD AND COMPUTATIONAL DETAILS}

\section{A. The WB-AIMP Hamiltonian}

The Dirac equation for the large radial components of one electron in a central field ${ }^{9}$ has been taken as the basis of approximate relativistic atomic and molecular computational methods by Cowan and Griffin ${ }^{23}$ (spin-free) and Wood and Boring $^{10}$ (spin-orbit) after neglecting the small component contributions and imposing boundary conditions at the nucleus. Katsuki and Huzinaga ${ }^{7,8}$ proposed to transfer Wood-Boring's approach to core model potential calculations. A practical implementation of these ideas resulted in the WB-AIMP method which is described in Ref. 6. Here, we briefly summarize these works.

The WB-AIMP Hamiltonian for a molecule reads

$$
\begin{aligned}
H^{\mathrm{WB}-\mathrm{AIMP}}= & H^{\mathrm{CG}-\mathrm{AIMP}} \\
& +\sum_{i}^{\text {val.elec. atoms val.orb. }} \sum_{I} \sum_{n l} V_{S O, n l}^{I, M P}\left(r_{i}\right) \hat{O}_{l}^{I} \hat{\boldsymbol{l}}^{I} \hat{\boldsymbol{s}} \hat{O}_{l}^{I} .
\end{aligned}
$$

Here $H^{\text {CG-AIMP }}$ (fully described in Ref. 6) is the spin-free many-electron Hamiltonian of the Cowan-Griffin based relativistic $a b$ initio core model potential approach; this is a relativistic version of the AIMP method, ${ }^{24}$ in which the core potentials are obtained directly from the core orbitals without resorting to parametrization procedures, thus eliminating the basis set dependences present in older versions. The second term is the spin-orbit operator, which is a sum of oneelectron $(i)$ atomic $(I)$ effective spin-orbit operators of the form proposed by Pitzer and Winter. ${ }^{4}$ Herein, $n l$ runs over the valence orbitals of each atom, $\hat{l}^{I}$ and $\hat{s}$ are the usual vector angular momentum and spin operators, respectively, and the projectors $\hat{O}_{l}^{I}$ are defined as $\hat{O}_{l}^{I}$ $=\Sigma_{m=-l}^{+l}|l m, I\rangle\langle l m, I|$. In the spin-orbit potential

$$
V_{S O, n l}^{I, M P}\left(r_{i}\right)=\lambda^{I} \sum_{k} \frac{B_{k}^{n l, I} \exp \left(-\beta_{k}^{n l, I} r_{i}^{2}\right)}{r_{i}^{2}}
$$

we have set the atomic scaling factor $\lambda^{I}$ (Ref. 6) to 1 , and the parameters $\left\{B_{k}^{n l, I}, \beta_{k}^{n l, I}\right\}$ are determined through weighted least-squares fitting to the radial part of the Wood-Boring spin-orbit operator ${ }^{10}$ which reads

$$
V_{S O, n l}(r)=\frac{\alpha^{2}}{\left(2+\alpha^{2}\left[\epsilon_{n l}-V(r)\right]\right) r} \frac{d V(r)}{d r} .
$$

Herein, $\alpha$ is the fine-structure constant, $\boldsymbol{\epsilon}_{n l}$ are the orbital energies of the spin-free relativistic equations of Cowan and Griffin, and $V(r)$ is an $X \alpha$ approximation to the HartreeFock one-electron potential. ${ }^{23}$ This effective one-electron spin-orbit operator includes an average of two-electron contributions through the use of the $X \alpha$ Hartree-Fock potential $V(r)$, although its detailed relationship to a mean-field spinorbit operator is unknown. In an attempt to correct for possible two-electron contribution deficiencies, $\lambda^{I}$ has previously been used as an empirical parameter, ${ }^{6}$ but we assigned unity to it within this paper.

The spin-dependent $H^{\text {WB-AIMP }}$ Hamiltonian is used in spin-orbit CI calculations in a basis of double-group symmetry-adapted functions, with HF or CASSCF orbitals produced with the spin-free $H^{\text {CG-AIMP }}$ Hamiltonian.

\section{B. The spin-free-state-shifted Hamiltonian}

The spin-free-state-shifted technique $(s f s s)$ was introduced in Ref. 22 as a means of simplifying spin-orbit CI calculations by decoupling correlation and spin-orbit effects, in the line of previous works. ${ }^{3,20,21,25}$ The idea behind this is the following: The size of the spin-orbit splittings is governed by the size of the spin-orbit couplings between the spin-free states $\Phi\left(i S M_{S} \Gamma \gamma\right)$ of a given system and their energy differences. Herein, $i$ denotes an ordinal number, $S$, $M_{S}$ the spin quantum numbers, and $\Gamma, \gamma$ the spatial point group irreducible representation and subspecies, respec- 
tively. However, spin-orbit coupling and excitation energies demand different degrees of quality in the description of the wavefunctions. In particular, a relatively small CI space $\mathscr{P}$ is often good enough for the calculation of the spin-orbit interaction, since the spin-orbit coupling operator can, to a good approximation, be represented by effective one-electron terms,${ }^{26}$ and therefore singly excited configurations make the major contributions to the spin-orbit matrix elements. A good description of the spin-free electronic spectrum requires, on the other hand, an accurate description of electron correlation effects and also a much larger CI space, say $\mathscr{G}$. Let us call $\Phi^{\mathscr{G}}\left(i S M_{S} \Gamma \gamma\right)$ the spin-free CI wave functions meeting the latter requirements and $E^{\mathscr{G}}(i S \Gamma)$ their corresponding eigenvalues while $\Phi^{\mathscr{P}}\left(i S M_{S} \Gamma \gamma\right)$ and $E^{\mathscr{P}}(i S \Gamma)$ denote the eigenvectors and eigenvalues, respectively, of the small CI space $\mathscr{P}$. Under these circumstances, it is reasonable to use the smaller CI space $\mathscr{P}$ to calculate the spin-orbit couplings and the larger CI space $\mathscr{G}$ to calculate the spin-free energy differences. In spin-orbit CI calculations, this can be achieved in a simple way by using the smaller CI space $\mathscr{P}$ and a spin-free-state-shifted spin-orbit Hamiltonian, $H_{s f s S}^{S O}$, which is related to a normal spin-orbit Hamiltonian, $H^{S O}$ [i.e., $H^{\text {WB-AIMP }}$ in Eq. (1)], by

$$
\begin{aligned}
H_{s f s s}^{S O}= & H^{S O} \\
& +\sum_{i S M_{S} \Gamma \gamma} \delta(i S \Gamma)\left|\Phi^{\mathscr{P}}\left(i S M_{S} \Gamma \gamma\right)\right\rangle\left\langle\Phi^{\mathscr{P}}\left(i S M_{S} \Gamma \gamma\right)\right|,
\end{aligned}
$$

with the shifting constants $\delta(i S \Gamma)$ defined by

$$
\begin{aligned}
\delta(i S \Gamma)= & {\left[E^{\mathscr{Q}}(i S \Gamma)-E^{\mathscr{Q}}(\text { G.S. })\right]-\left[E^{\mathscr{P}}(i S \Gamma)\right.} \\
& \left.-E^{\mathscr{P}}(\text { G.S. })\right] .
\end{aligned}
$$

Herein, $E^{\mathscr{G}}\left(\right.$ G.S.) and $E^{\mathscr{P}}(G . S$.) are the spin-free CI energies of a common given state (usually the ground state) corresponding to the $\mathscr{G}$ and $\mathscr{P} \mathrm{CI}$ spaces, respectively. The construction of the shifting operator [Eq. (4)] requires the knowledge of $E^{\mathscr{G}}(i S \Gamma)$ and $E^{\mathscr{G}}($ G.S.) which may be obtained by spin-free methods taking full advantage of the spin symmetry and therefore capable of handling much larger CI spaces $\mathscr{G}$ than feasible in a spin-orbit CI. Usually due to technical hindrances $\mathscr{G}$ is still of limited size thus neglecting a part of the correlation effects. Alternatively, the spectrum of $E^{\mathscr{G}}(i S \Gamma)$ may be taken from experimental data provided that spin-orbit effects are properly removed-which is possible for atomic systems. Experimental spin-free reference energies obtained by a simple statistical $J$-averaging procedure are often unreliable. In other words, in general one cannot rely on Landé's interval rule. Experimentalists identify the symmetries of terms by means of a general least-squares (GLS) fitting of an experimental spectrum. Such data for singly ionized iridium can be found in a publication by Kleef and Metsch. ${ }^{27}$ GLS fittings to the spectrum of singly ionized iridium were carried out by Kleef and Metsch themselves ${ }^{27}$ and by Wyart et al. ${ }^{28}$ The parameters used in such a model Hamiltonian are divided into parts strictly representing either pure spin-free or pure spin-dependent interactions. Most reliable spin-free reference energy differences can therefore be obtained by using all the optimized spin-free parameters while setting all the spin-dependent ones to zero. The empirical spin-free reference values used in this work have been obtained by Uylings ${ }^{29}$ imposing the above-mentioned procedure.

In practice, the sum in Eq. (4) must be limited, with the number of spin-free states depending on the particular problem; it seems reasonable to include all the states of interest and the ones above them within a chosen energy threshold. Since the calculated spin-orbit CI functions in the $\mathscr{P}$ space can be expressed as a sum of the spin-free functions $\Phi^{\mathscr{P}}\left(i S M_{S} \Gamma \gamma\right)$ and a residue, the norm of the residue can be employed as a criterion whether an appropriate number of spin-free states has been chosen or not.

\section{Details of the calculations}

We used relativistic core model potentials and valence basis sets from Ref. 19. The WB-AIMP's correspond to the $[C d, 4 f]$ frozen core of $\operatorname{Ir}\left(5 d^{7} 6 s^{2}-{ }^{4} \mathrm{~F}\right)$. A Gaussian valence basis set $(13 s 9 p 8 d)$ of general contraction was optimized in a spin-free CG-AIMP SCF calculation of $\operatorname{Ir}\left(5 d^{7} 6 s^{2}-{ }^{4} \mathrm{~F}\right)$ and was thereafter spin-orbit corrected according to the procedure described in detail in Ref. 6 . This basis set was valencesplit and further augmented by one $p$ polarization function (exponent 0.081 ) ${ }^{30}$ one additional $d$ primitive (exponent 0.0617 ) obtained by extrapolation from the outermost exponents, and two contracted $f$ functions. The five primitive $f$ functions and their contraction coefficients were determined by maximizing the overlap of a single contraction with the numerical $4 f$ core orbital. Finally the most diffuse $f$ primitive was decontracted resulting in a total of $3 s, 3 p, 4 d$, and $2 f$ contracted functions with contraction pattern $(11,1,1 /$ $8,1,1 / 6,1,1,1 / 4,1)$.

We performed all spin-free calculations with the MOLCAS-3 package. ${ }^{31}$ For several atomic states of the $(5 d, 6 s)^{8}$ manifold orbitals were optimized with the CGAIMP spin-free CASSCF method. ${ }^{32-34}$ By using $C_{i}$ symmetry and averaging over all the degenerate components of every term, atomic orbitals of true spherical symmetry were obtained. With these, we performed Averaged Coupled-Pair Functional (ACPF $)^{35,36}$ calculations, an approximatively size-consistent multireference $\mathrm{CI}(\mathrm{SD})$ procedure.

WB-AIMP spin-orbit CI calculations have been carried out in a basis of double-group symmetry-adapted functions with a modified version of the COLUMBUS suite of programs. ${ }^{37}$ We used $\bar{D}_{2 h}$ double-group symmetry but obtained degeneracies with energy separations below $10^{-6}$ hartrees for the components of a given $J$ quantum number. This greatly simplifies the assignment of angular momentum quantum numbers; in fact, diagonalizing only the $A_{g}$ and $B_{1 g}$ symmetry blocks is sufficient to identify all the gerade $J$ states. In the integral transformation step the highest $s, p, d$, and $f$ shells which exhibit core character are removed from the virtual space. Using this one-particle basis we carried out spin-orbit CI calculations in three different spaces of doublegroup symmetry adapted functions: the reference space $(5 d, 6 s)^{8}$ (ref-CI), the reference space plus single excitations $(\mathrm{CI}(\mathrm{S}))$, and the reference space plus single and double ex- 
TABLE I. Number of $\bar{D}_{2 h}$ double-group symmetry adapted functions used in the spin-orbit CI calculations. In parentheses, approximate CPU time [minutes] as measured on an IBM RS/6000 365 workstation.

\begin{tabular}{ccc}
\hline \hline & \multicolumn{2}{c}{ Symmetry block } \\
\cline { 2 - 3 } Calculation & $A_{g}$ & $B_{1 g}$ \\
\hline ref-CI & $135(\ll 1)$ & $120(\ll 1)$ \\
CI(S) & $4491(15)$ & $4476(15)$ \\
CI(SD) & $102097(2200)$ & $101622(2200)$ \\
\hline \hline
\end{tabular}

citations $(\mathrm{CI}(\mathrm{SD}))$. The $\mathrm{CI}$ expansion lengths are shown in Table I.

\section{RESULTS AND DISCUSSION}

Table II displays the results of the spin-free even spectrum of $\mathrm{Ir}^{+}$, in particular the terms associated with the configurations $5 d^{8}, 5 d^{7} 6 s^{1}$, and $5 d^{6} 6 s^{2}$. In the last column we have included what we call the empirical spin-free data. The columns labeled ACPF correspond to eight correlated electrons. In the correlation treatment three different sets of oneparticle bases were employed: In the first case, orbitals were optimized for each term, in the second $5 d^{8}{ }_{-}^{3} \mathrm{~F}$ orbitals were used for all terms, and finally in the third case $5 d^{7} 6 s^{1}-5 \mathrm{~F}$ orbitals were employed throughout. Only a small dependency on the choice of orbitals is observed; single excitations are obviously sufficient to account for orbital relaxation effects at the spin-free level. We will therefore use atomic orbitals optimized for $5 d^{7} 6 s^{1}-5 \mathrm{~F}$ in the remaining calculations of this paper. The deviations from the empirical energies are of the order to be expected for the kind of basis set and correlation treatment employed. The results of the CI calculations either with a reference-CI space of $(5 d, 6 s)^{8}$ only, ref-CI, with the addition of single excitations, $\mathrm{CI}(\mathrm{S})$, or with single and double excitations, $\mathrm{CI}(\mathrm{SD})$, are given in this table as well; they constitute the set of $E^{\mathscr{P}}(i S \Gamma)$ necessary for defining the energy shifts according to Eq. (5). Obviously, already the single excitations enhance the quality of

TABLE II. Ab initio and empirically calculated energies $[\mathrm{eV}]$ of the lowest spin-free terms of $\mathrm{Ir}^{+}$. See text for a description.

\begin{tabular}{|c|c|c|c|c|c|c|c|c|}
\hline \multirow{3}{*}{$\begin{array}{l}\text { Main } \\
\text { conf. }\end{array}$} & \multirow[b]{3}{*}{ Term } & \multicolumn{3}{|c|}{$\mathrm{ACPF}$} & & & & \multirow[b]{3}{*}{ Empirical } \\
\hline & & \multicolumn{3}{|c|}{ with AO's optimized for } & \multicolumn{3}{|c|}{ CI with ${ }^{5} \mathrm{~F}$ AO's } & \\
\hline & & each term & ${ }^{3} \mathrm{~F}$ & ${ }^{5} \mathrm{~F}$ & ref-CI & $\mathrm{CI}(\mathrm{S})$ & $\mathrm{CI}(\mathrm{SD})$ & \\
\hline$\overline{d^{7} s^{1}}$ & ${ }^{5} \mathrm{~F}$ & 0.0 & 0.0 & 0.0 & 0.0 & 0.0 & 0.0 & 0.0 \\
\hline$d^{8}$ & ${ }^{3} \mathrm{~F}$ & 0.035 & 0.034 & 0.048 & 1.051 & 0.323 & 0.129 & 0.088 \\
\hline$d^{8}$ & ${ }^{3} \mathrm{P}$ & 0.488 & 0.494 & 0.495 & 1.211 & 0.693 & 0.558 & 0.342 \\
\hline$d^{8}$ & ${ }^{1} \mathrm{D}$ & 0.824 & 0.825 & 0.839 & 1.739 & 1.122 & 0.926 & 0.693 \\
\hline$d^{7} s^{1}$ & ${ }^{5} \mathrm{P}$ & 1.328 & 1.367 & 1.328 & 1.506 & 1.414 & 1.336 & 1.068 \\
\hline$d^{8}$ & ${ }^{1} \mathrm{G}$ & 1.489 & 1.493 & 1.501 & 2.179 & 1.663 & 1.555 & 1.189 \\
\hline$d^{7} s^{1}$ & $b^{3} \mathrm{~F}$ & 1.542 & n.conv. & 1.554 & 1.818 & 1.617 & 1.584 & 1.374 \\
\hline$d^{7} s^{1}$ & ${ }^{3} \mathrm{G}$ & - & - & 1.968 & 2.018 & 1.997 & 1.983 & 1.649 \\
\hline$d^{7} s^{1}$ & $b^{3} \mathrm{P}$ & - & - & 2.465 & 2.873 & 2.553 & 2.486 & 2.000 \\
\hline$d^{7} s^{1}$ & ${ }^{3} \mathrm{H}$ & - & - & 2.548 & 2.555 & 2.516 & 2.539 & 2.073 \\
\hline$d^{7} s^{1}$ & ${ }^{3} \mathrm{D}$ & - & - & 2.473 & 2.687 & 2.632 & 2.508 & 2.146 \\
\hline$d^{6} s^{2}$ & ${ }^{5} \mathrm{D}$ & 2.669 & 2.931 & 2.770 & 3.191 & 2.494 & 2.742 & 2.171 \\
\hline$d^{7} s^{1}$ & ${ }^{1} \mathrm{P}$ & & & & & 3.455 & & 2.832 \\
\hline$d^{7} s^{1}$ & $c^{3} \mathrm{P}$ & & & & & 3.452 & & 2.834 \\
\hline$d^{7} s^{1}$ & $b^{1} \mathrm{G}$ & & & & & 3.531 & & 2.836 \\
\hline$d^{7} s^{1}$ & ${ }^{1} \mathrm{H}$ & & & & & 3.468 & & 2.896 \\
\hline$d^{7} s^{1}$ & $b^{1} \mathrm{D}$ & & & & & 3.747 & & 3.006 \\
\hline$d^{7} s^{1}$ & $c^{3} \mathrm{~F}$ & & & & & 3.901 & & 3.086 \\
\hline$d^{7} s^{1}$ & ${ }^{1} \mathrm{~F}$ & & & & & 4.349 & & 3.447 \\
\hline$d^{8}$ & ${ }^{1} \mathrm{~S}$ & & & & & 4.763 & & 3.859 \\
\hline$d^{6} s^{2}$ & $b^{3} \mathrm{H}$ & & & & & 4.731 & & 3.987 \\
\hline$d^{6} s^{2}$ & $b^{3} \mathrm{G}$ & & & & & & & 4.424 \\
\hline$d^{6} s^{2}$ & $d^{3} \mathrm{~F}$ & & & & & & & 4.546 \\
\hline$d^{6} s^{2}$ & $d^{3} \mathrm{P}$ & & & & & & & 4.725 \\
\hline$d^{7} s^{1}$ & $b^{3} \mathrm{D}$ & & & & & & & 4.738 \\
\hline$d^{6} s^{2}$ & ${ }^{1} \mathrm{I}$ & & & & & & & 4.802 \\
\hline$d^{7} s^{1}$ & $c^{1} \mathrm{D}$ & & & & & & & 4.968 \\
\hline$d^{6} s^{2}$ & $c^{1} \mathrm{G}$ & & & & & & & 5.062 \\
\hline$d^{6} s^{2}$ & $c^{3} \mathrm{D}$ & & & & & & & 5.277 \\
\hline$d^{6} s^{2}$ & $b^{1} \mathrm{~S}$ & & & & & & & 5.513 \\
\hline$d^{6} s^{2}$ & $b^{1} \mathrm{~F}$ & & & & & & & 6.514 \\
\hline$d^{6} s^{2}$ & $d^{1} \mathrm{D}$ & & & & & & & 6.755 \\
\hline$d^{6} s^{2}$ & $e^{3} \mathrm{~F}$ & & & & & & & 6.841 \\
\hline$d^{6} s^{2}$ & $d^{1} \mathrm{G}$ & & & & & & & 7.536 \\
\hline$d^{6} s^{2}$ & $e^{3} \mathrm{P}$ & & & & & & & 7.900 \\
\hline$d^{6} s^{2}$ & $e^{1} \mathrm{D}$ & & & & & & & 9.299 \\
\hline$d^{6} s^{2}$ & $c^{1} \mathrm{~S}$ & & & & & & & 11.421 \\
\hline
\end{tabular}


TABLE III. Spin-orbit CI and experimental energies [eV] of the lowest even states of $\mathrm{Ir}^{+}$.

\begin{tabular}{|c|c|c|c|c|c|c|c|c|c|}
\hline \multirow[b]{2}{*}{$\mathrm{J}$} & \multirow{2}{*}{$\begin{array}{l}\text { Main } \\
\text { term }\end{array}$} & \multirow[b]{2}{*}{$\mathrm{CI}(\mathrm{SD})$} & \multicolumn{3}{|c|}{ ACPF-sfss ${ }^{\text {a }}$} & \multicolumn{3}{|c|}{ empirical-sfss ${ }^{\mathrm{b}}$} & \multirow[b]{2}{*}{ Experiment } \\
\hline & & & ref-CI & $\mathrm{CI}(\mathrm{S})$ & $\mathrm{CI}(\mathrm{SD})$ & ref-CI & $\mathrm{CI}(\mathrm{S})$ & $\mathrm{CI}(\mathrm{SD})$ & \\
\hline 5 & ${ }^{5} \mathrm{~F}$ & 0.0 & 0.0 & 0.0 & 0.0 & 0.0 & $0.0(0.0)$ & 0.0 & 0.0 \\
\hline 4 & ${ }^{3} \mathrm{~F}$ & 0.320 & 0.244 & 0.261 & 0.243 & 0.302 & $0.317(0.314)$ & 0.299 & 0.281 \\
\hline 2 & ${ }^{3} \mathrm{P}$ & 0.566 & 0.470 & 0.496 & 0.502 & 0.343 & $0.373(0.371)$ & 0.382 & 0.383 \\
\hline 4 & ${ }^{5} \mathrm{~F}$ & 0.626 & 0.617 & 0.628 & 0.624 & 0.598 & $0.614(0.609)$ & 0.610 & 0.594 \\
\hline 3 & ${ }^{5} \mathrm{~F}$ & 1.073 & 1.079 & 1.071 & 1.064 & 1.080 & $1.073(1.067)$ & 1.062 & 1.015 \\
\hline 2 & & 1.225 & 1.198 & 1.204 & 1.195 & 1.149 & 1.159 (1.144) & 1.147 & 1.113 \\
\hline 1 & ${ }^{3} \mathrm{P}$ & 1.306 & 1.243 & 1.280 & 1.277 & 1.118 & $1.165(1.137)$ & 1.167 & 1.124 \\
\hline 3 & ${ }^{3} \mathrm{~F}$ & 1.295 & 1.252 & 1.220 & 1.224 & 1.313 & $1.279(1.280)$ & 1.286 & 1.231 \\
\hline 0 & ${ }^{3} \mathrm{P}$ & 1.635 & 1.704 & 1.611 & 1.580 & 1.567 & $1.483(1.426)$ & 1.455 & 1.390 \\
\hline 2 & & 1.533 & 1.534 & 1.501 & 1.492 & 1.504 & $1.473(1.460)$ & 1.462 & 1.402 \\
\hline 4 & & 1.656 & 1.589 & 1.630 & 1.631 & 1.434 & $1.481(1.461)$ & 1.485 & 1.453 \\
\hline 1 & ${ }^{5} \mathrm{~F}$ & 1.627 & 1.620 & 1.608 & 1.601 & 1.566 & $1.547(1.546)$ & 1.539 & 1.483 \\
\hline 3 & ${ }^{5} \mathrm{P}$ & 1.838 & 1.857 & 1.845 & 1.829 & 1.645 & $1.637(1.636)$ & 1.621 & 1.576 \\
\hline 2 & ${ }^{5} \mathrm{P}$ & 2.228 & 2.210 & 2.196 & 2.197 & 2.122 & $2.070(2.019)$ & 2.063 & 1.944 \\
\hline 4 & & 2.563 & 2.538 & 2.526 & 2.528 & 2.253 & $2.224(2.198)$ & 2.233 & 2.134 \\
\hline 2 & & 2.449 & 2.420 & 2.389 & 2.402 & 2.232 & $2.247(2.205)$ & 2.279 & 2.159 \\
\hline 5 & ${ }^{3} \mathrm{G}$ & 2.511 & 2.519 & 2.509 & 2.503 & 2.237 & $2.231(2.215)$ & 2.225 & 2.167 \\
\hline 3 & $b^{3} \mathrm{~F}$ & 2.442 & 2.440 & 2.422 & 2.417 & 2.249 & $2.231(2.226)$ & 2.226 & 2.170 \\
\hline 1 & ${ }^{5} \mathrm{P}$ & 2.645 & 2.767 & 2.661 & 2.630 & 2.556 & $2.458(2.393)$ & 2.435 & 2.316 \\
\hline 2 & & 2.778 & 3.014 & 2.813 & 2.758 & 2.788 & $2.576(2.468)$ & 2.522 & 2.349 \\
\hline 4 & ${ }^{5} \mathrm{D}$ & 2.905 & 2.951 & 2.918 & 2.922 & 2.525 & $2.489(2.475)$ & 2.478 & 2.390 \\
\hline 4 & ${ }^{3} \mathrm{G}$ & 2.897 & 3.022 & 2.900 & 2.884 & 2.693 & $2.616(2.586)$ & 2.600 & 2.516 \\
\hline 1 & $b^{3} \mathrm{P}$ & 2.958 & 3.056 & 3.002 & 2.948 & 2.763 & $2.733(2.581)$ & 2.703 & 2.534 \\
\hline 6 & ${ }^{3} \mathrm{H}$ & 3.251 & 3.273 & 3.262 & 3.261 & 2.831 & $2.820(2.821)$ & 2.819 & 2.761 \\
\hline 2 & & 3.161 & 3.287 & 3.165 & 3.128 & 3.030 & $2.948(2.889)$ & 2.921 & 2.786 \\
\hline 3 & ${ }^{3} \mathrm{G}$ & 3.275 & 3.287 & 3.272 & 3.261 & 3.009 & $2.993(2.952)$ & 2.989 & 2.876 \\
\hline 3 & ${ }^{5} \mathrm{D}$ & 3.533 & 3.586 & 3.556 & 3.556 & 3.075 & $3.063(3.030)$ & 3.051 & 2.942 \\
\hline 0 & $b^{3} \mathrm{P}$ & 3.568 & 3.644 & 3.576 & 3.561 & 3.209 & $3.165(3.081)$ & 3.177 & $3.059^{\mathrm{c}}$ \\
\hline 5 & ${ }^{3} \mathrm{H}$ & 3.603 & 3.634 & 3.611 & 3.610 & 3.282 & $3.262(3.167)$ & 3.263 & 3.101 \\
\hline 1 & & 3.649 & 3.784 & 3.657 & 3.640 & 3.450 & $3.376(3.280)$ & 3.340 & $3.109^{c}$ \\
\hline 2 & ${ }^{5} \mathrm{D}$ & 3.728 & 3.879 & 3.760 & 3.731 & 3.388 & $3.351(3.336)$ & 3.326 & 3.170 \\
\hline 4 & ${ }^{3} \mathrm{H}$ & 3.792 & 4.015 & 3.806 & 3.793 & 3.660 & $3.522(3.339)$ & 3.515 & $3.248^{c}$ \\
\hline 3 & ${ }^{3} \mathrm{D}$ & 3.642 & 3.635 & 3.626 & 3.617 & 3.388 & $3.381(3.379)$ & 3.374 & 3.272 \\
\hline 2 & & 3.886 & 3.961 & 3.900 & 3.880 & 3.762 & $3.644(3.547)$ & 3.622 & $3.425^{\mathrm{c}}$ \\
\hline 0 & ${ }^{5} \mathrm{D}$ & 4.032 & 4.292 & 4.174 & 4.050 & 3.835 & $3.790(3.628)$ & 3.709 & $3.429^{c}$ \\
\hline 1 & & 4.105 & 4.164 & 4.144 & 4.118 & 3.720 & $3.705(3.697)$ & 3.681 & 3.546 \\
\hline
\end{tabular}

${ }^{a}$ Spin-free-state-shifted Hamiltonian with shifting parameters obtained from spin-free ACPF calculations (fifth column in Table II). Spin-free states up to ${ }^{5} \mathrm{D}$ have been included in the shifting operator.

${ }^{\mathrm{b}}$ Spin-free-state-shifted Hamiltonian with shifting parameters obtained from spin-free empirical data (last column in Table II). Spin-free states up to ${ }^{5} \mathrm{D}$ have been included in the shifting operator; the numbers in parentheses correspond to including spin-free states up to $b^{3} \mathrm{H}$.

${ }^{\mathrm{c}}$ Reference 27. Result predicted by fitting of experimentally observed lines. The fitted lines show a deviation of the order of $0.01 \mathrm{eV}$ with respect to the observed ones.

the results which is furthermore improved by double excitations. The $\mathrm{CI}(\mathrm{SD})$ calculation leads to a spin-free spectrum quite close to the corresponding ACPF calculation; this might be expected because only eight electrons are correlated and size-consistency effects are concomitantly small.

The results of the spin-orbit CI calculations are given in Table III and Fig. 1 accompanied by the experimental measurements. ${ }^{27}$ The plain spin-orbit $\mathrm{CI}(\mathrm{SD})$ results-which do not include any shifting-reveal a reasonable agreement with experiment, approximately of the quality reached by four-component DHF+CI methods in similar systems if only the valence electrons are correlated. ${ }^{2}$ Similar observations were made previously in other cases. ${ }^{6,18}$

The spin-orbit CI calculations with spin-free states shifted to the ACPF excitation energies (fifth column in Table II), while including all terms of Eq. (4) up to ${ }^{5} \mathrm{D}$, are presented in the columns labeled ACPF-sfss. The ACPF-sfss
$\mathrm{CI}(\mathrm{SD})$ results are comparable to the ones from plain $\mathrm{CI}(\mathrm{SD})$ calculations. However, if the $s f s s$ technique is used, single excitations seem to be sufficient to achieve the same degree of improvement [ACPF-sfss $\mathrm{CI}(\mathrm{S})$ results]. This means that the correlation effects have already been dealt with at the spin-free ACPF level and the subsequent spin-orbit CI(S) calculation takes care of only the spin-orbit interactions, including partially spin-orbit polarization effects; if spin-orbit polarization is missing (ACPF-sfss ref-CI) the results are poorer, although not too much. The CI(SD) spin-orbit splittings are systematically overestimated when compared with experiment. Since they depend not only on the size of the spin-orbit couplings but also on the relative energies of the spin-free terms - and these are far from being realistic (Table II) mainly due to an insufficient treatment of the electron correlation effects-it is not clear at this point how much of 


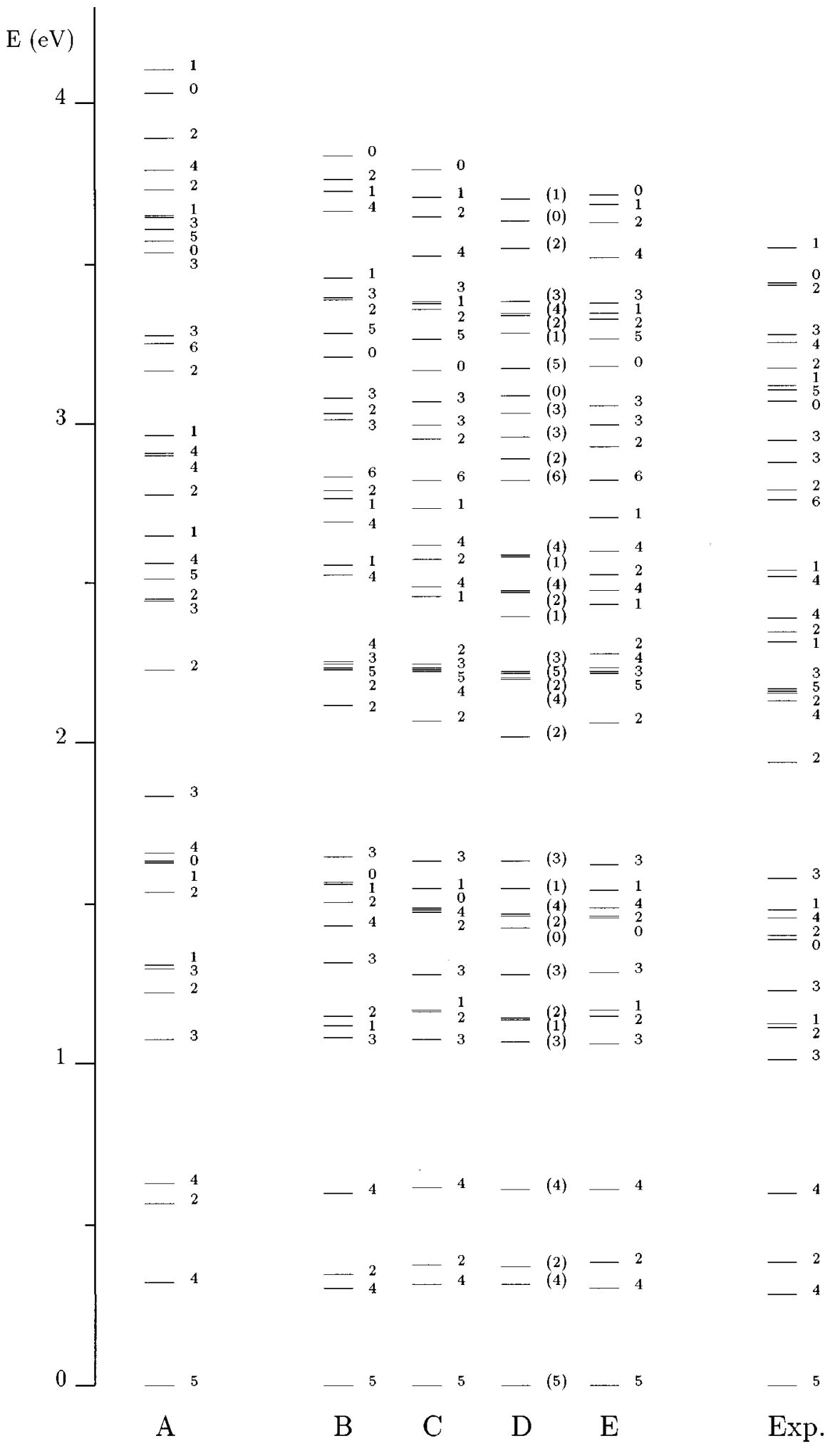

FIG. 1. Even spectrum of $\mathrm{Ir}^{+}$. A: Spin-orbit CI(SD) calculation of Table III. B, C, D, E: Empirically spin-free-state-shifted spin-orbit CI calculations as in Table III; B, C, and $\mathbf{E}$ are ref-CI, CI(S), and CI(SD) calculations, respectively, with shifting terms up to ${ }^{5} \mathrm{D}$ in Table II; D is a CI(S) calculation with shifting terms up to $b^{3} \mathrm{H}$ according to Table II.

the overestimation is due to this and how much is due to deficiencies in the spin-orbit method in use.

In this respect, we can isolate the contributions of the spin-orbit operator by performing spin-orbit CI calculations with spin-free states shifted to the empirical spin-free results (last column in Table II). The values corresponding to an inclusion of the terms up to ${ }^{5} \mathrm{D}$ in Eq. (4) are presented in Table III as well as in Fig. 1. The empirical-sfss ref-CI calculation (B in Fig. 1) which does not include spin-orbit polarization, already brings about significant improvements 
TABLE IV. Analysis of the contributions [\%] of the spin-free states to the spin-orbit states of $\operatorname{Ir}^{+}$in the empirical-sfss CI(S) calculations of Table III. See footnote $\mathrm{b}$ in Table III.

\begin{tabular}{|c|c|c|c|c|c|}
\hline \multirow{2}{*}{$\frac{J}{5}$} & \multirow[t]{2}{*}{ Contributions } & \multicolumn{2}{|c|}{ Sum } & \multicolumn{2}{|c|}{$\begin{array}{l}\text { Deviation from } \\
\text { experiment }[\mathrm{eV}]\end{array}$} \\
\hline & & 100 & $(100)$ & 0.0 & $(0.0)$ \\
\hline 4 & $94(94){ }^{3} \mathrm{~F}+4(4){ }^{1} \mathrm{G}$ & 100 & (99) & 0.036 & $(0.033)$ \\
\hline 2 & $62(62){ }^{3} \mathrm{P}+24(23){ }^{1} \mathrm{D}+6(7){ }^{5} \mathrm{P}$ & 99 & $(100)$ & -0.010 & $(-0.012)$ \\
\hline 4 & $77(76){ }^{5} \mathrm{~F}+11(12) b^{3} \mathrm{~F}+8(8){ }^{3} \mathrm{G}$ & 99 & $(100)$ & 0.004 & $(0.015)$ \\
\hline 3 & $75(75){ }^{5} \mathrm{~F}+9(9){ }^{3} \mathrm{D}+8(8){ }^{3} \mathrm{~F}+4(4){ }^{5} \mathrm{P}$ & 98 & (99) & 0.058 & $(0.052)$ \\
\hline 2 & $36(34){ }^{5} \mathrm{~F}+21(21){ }^{1} \mathrm{D}+19(19){ }^{3} \mathrm{~F}+12(12)^{3} \mathrm{D}+5(4){ }^{3} \mathrm{P}$ & 97 & (99) & 0.046 & $(0.031)$ \\
\hline 1 & $\left.54(51){ }^{3} \mathrm{P}+19(19)\right)^{5} \mathrm{~F}+13(14){ }^{3} \mathrm{D}+8(8){ }^{5} \mathrm{P}+(4){ }^{1} \mathrm{P}$ & 96 & $(100)$ & 0.041 & $(0.013)$ \\
\hline 3 & $90(90){ }^{3} \mathrm{~F}+7(7){ }^{5} \mathrm{~F}$ & 100 & $(100)$ & 0.048 & $(0.049)$ \\
\hline 0 & $89(87){ }^{3} \mathrm{P}+4(3) b^{3} \mathrm{P}+(8){ }^{1} \mathrm{~S}$ & 95 & $(100)$ & 0.093 & $(0.036)$ \\
\hline \multirow[t]{2}{*}{2} & $42(43){ }^{5} \mathrm{~F}+19(18){ }^{3} \mathrm{~F}+13(13){ }^{5} \mathrm{P}+12(11){ }^{1} \mathrm{D}+8(7){ }^{3} \mathrm{P}$ & & & & \\
\hline & $+4(5)^{3} \mathrm{D}$ & 98 & $(100)$ & 0.071 & $(0.058)$ \\
\hline 4 & $50(49) b^{3} \mathrm{~F}+19(20){ }^{5} \mathrm{~F}+18(17){ }^{1} \mathrm{G}+4(4){ }^{3} \mathrm{G}+(4) c^{3} \mathrm{P}$ & 97 & $(100)$ & 0.028 & $(0.008)$ \\
\hline 1 & $57(56){ }^{5} \mathrm{~F}+24(26){ }^{3} \mathrm{P}+10(10){ }^{3} \mathrm{D}+7(7){ }^{5} \mathrm{P}$ & 99 & $(100)$ & 0.064 & $(0.063)$ \\
\hline 3 & $79(78){ }^{5} \mathrm{P}+7(7){ }^{5} \mathrm{~F}+5(6){ }^{3} \mathrm{D}+5(6) b^{3} \mathrm{~F}$ & 98 & (98) & 0.061 & $(0.060)$ \\
\hline 2 & $60(56){ }^{5} \mathrm{P}+16(16){ }^{3} \mathrm{~F}+5(8){ }^{3} \mathrm{P}+4 b^{3} \mathrm{P}+(10) b^{1} \mathrm{G}$ & 93 & (99) & 0.116 & $(0.075)$ \\
\hline 4 & $40(38){ }^{1} \mathrm{G}+32(36){ }^{5} \mathrm{D}+15(13) b^{3} \mathrm{~F}++6(5){ }^{3} \mathrm{H}+(5) c^{3} \mathrm{~F}$ & 95 & (97) & 0.090 & $(0.064)$ \\
\hline \multirow[t]{2}{*}{2} & $34(31){ }^{3} \mathrm{~F}+23(15) b^{3} \mathrm{P}+19(20){ }^{1} \mathrm{D}+8(14) b^{3} \mathrm{~F}+4(5){ }^{3} \mathrm{P}$ & & & & \\
\hline & $+(8) b^{1} \mathrm{D}$ & 95 & $(100)$ & 0.088 & $(0.046)$ \\
\hline 5 & $66(66){ }^{3} \mathrm{G}+22(20){ }^{3} \mathrm{H}+10(10){ }^{5} \mathrm{~F}+(4){ }^{1} \mathrm{H}$ & 98 & $(100)$ & 0.064 & $(0.048)$ \\
\hline 3 & $62(62) b^{3} \mathrm{~F}+15(14){ }^{3} \mathrm{D}+11(11){ }^{5} \mathrm{P}+9(9){ }^{3} \mathrm{G}$ & 99 & $(100)$ & 0.061 & $(0.056)$ \\
\hline 1 & $72(52){ }^{5} \mathrm{P}+16(19){ }^{3} \mathrm{P}+(9) b^{3} \mathrm{P}+(11) b^{1} \mathrm{G}+(5){ }^{1} \mathrm{P}$ & 94 & (99) & 0.142 & $(0.077)$ \\
\hline \multirow[t]{2}{*}{2} & $33(36) b^{3} \mathrm{P}+26(22) b^{3} \mathrm{~F}+10(6){ }^{3} \mathrm{P}+6(8){ }^{3} \mathrm{D}+4(4){ }^{5} \mathrm{D}$ & & & & \\
\hline & $+(11) b^{1} \mathrm{G}+(7) b^{1} \mathrm{D}$ & 86 & (98) & 0.227 & $(0.119)$ \\
\hline 4 & $56(48){ }^{5} \mathrm{D}+18(21){ }^{1} \mathrm{G}+15(17) b^{3} \mathrm{~F}$ & 94 & $(95)$ & 0.099 & $(0.085)$ \\
\hline 4 & $58(55){ }^{3} \mathrm{G}+17(17){ }^{3} \mathrm{H}+14(14){ }^{1} \mathrm{G}+5(5) b^{3} \mathrm{~F}$ & 96 & $(100)$ & 0.100 & $(0.070)$ \\
\hline 1 & $60(38) b^{3} \mathrm{P}+8(25){ }^{5} \mathrm{P}+6{ }^{5} \mathrm{D}+(6){ }^{5} \mathrm{~F}+(26){ }^{1} \mathrm{P}$ & 79 & (99) & 0.199 & $(0.047)$ \\
\hline 6 & $100(100){ }^{3} \mathrm{H}$ & 100 & $(100)$ & 0.059 & $(0.060)$ \\
\hline \multirow[t]{2}{*}{2} & $31(18) b^{3} \mathrm{~F}+18(22) b^{3} \mathrm{P}+16(17){ }^{1} \mathrm{D}+11(6){ }^{3} \mathrm{D}+6(7){ }^{3} \mathrm{~F}$ & & & & \\
\hline & $+4(7){ }^{5} \mathrm{P}+(5){ }^{3} \mathrm{P}+(5) b^{1} \mathrm{D}+(5) c^{3} \mathrm{~F}$ & 92 & (98) & 0.162 & $(0.103)$ \\
\hline 3 & $53(73){ }^{3} \mathrm{G}+34(4){ }^{5} \mathrm{D}+6(8){ }^{3} \mathrm{D}+(6){ }^{1} \mathrm{~F}$ & 96 & $(100)$ & 0.117 & $(0.076)$ \\
\hline 3 & $58(86){ }^{5} \mathrm{D}+25{ }^{3} \mathrm{G}+5(4) b^{3} \mathrm{~F}+4{ }^{3} \mathrm{D}+(4) c^{3} \mathrm{~F}$ & 94 & (97) & 0.121 & $(0.088)$ \\
\hline 0 & $61(58) b^{3} \mathrm{P}+31(19){ }^{5} \mathrm{D}+(18) b^{1} \mathrm{G}$ & 92 & (99) & 0.106 & $(0.022)$ \\
\hline 5 & $72(66){ }^{3} \mathrm{H}+15(9){ }^{3} \mathrm{G}+(23){ }^{1} \mathrm{H}$ & 88 & $(100)$ & 0.161 & $(0.066)$ \\
\hline \multirow[t]{2}{*}{1} & $31(30){ }^{5} \mathrm{D}+29(22){ }^{3} \mathrm{D}+11(10){ }^{5} \mathrm{~F}+5(17) b^{3} \mathrm{P}+(7){ }^{1} \mathrm{P}$ & & & & \\
\hline & $+(7) b^{1} \mathrm{G}$ & 80 & (96) & 0.267 & $(0.171)$ \\
\hline 2 & $76(71){ }^{5} \mathrm{D}+9(5) b^{3} \mathrm{P}+(4){ }^{3} \mathrm{D}$ & 89 & $(90)$ & 0.181 & $(0.166)$ \\
\hline 4 & $53(31){ }^{3} \mathrm{H}+24(18){ }^{3} \mathrm{G}+(4){ }^{5} \mathrm{D}+(29) c^{3} \mathrm{P}+(10) c^{3} \mathrm{~F}$ & 82 & (98) & 0.274 & $(0.091)$ \\
\hline 3 & $59(61){ }^{3} \mathrm{D}+26(25) b^{3} \mathrm{~F}+6(6){ }^{5} \mathrm{~F}+6(4){ }^{3} \mathrm{G}$ & 99 & (99) & 0.109 & $(0.107)$ \\
\hline \multirow[t]{2}{*}{2} & $48(37){ }^{3} \mathrm{D}+16(11) b^{3} \mathrm{~F}+11(10)^{5} \mathrm{~F}+7(12) b^{3} \mathrm{P}(6){ }^{5} \mathrm{D}$ & & & & \\
\hline & $+(10) b^{1} \mathrm{G}+(4) b^{1} \mathrm{D}+(4) c^{3} \mathrm{~F}$ & 88 & (98) & 0.219 & $(0.122)$ \\
\hline 0 & $57(56){ }^{5} \mathrm{D}+21 b^{3} \mathrm{P}+(31) b^{1} \mathrm{G}+(5){ }^{1} \mathrm{~S}$ & 81 & (93) & 0.361 & $(0.199)$ \\
\hline 1 & $51(55){ }^{5} \mathrm{D}+19(14){ }^{3} \mathrm{D}+17(17) b^{3} \mathrm{P}+6(5){ }^{5} \mathrm{~F}$ & 93 & (94) & 0.159 & $(0.151)$ \\
\hline
\end{tabular}

over the plain $\mathrm{CI}(\mathrm{SD})$ calculation (A in Fig. 1) and yields good spin-orbit splittings, thus pointing out the high quality of the spin-orbit operator in use. A partial addition of spinorbit polarization effects through single excitations [empirical-sfss $\mathrm{CI}(\mathrm{S}), \mathbf{C}$ in Fig. 1] refines the results, correcting some of the relative positions of the states, and finally leading to a very good spectrum. Addition of double excitations [empirical-sfss $\mathrm{CI}(\mathrm{SD}), \mathbf{E}$ in Fig. 1] does not significantly improve the results, in spite of its much larger cost: This is a consequence of the correlation effects on the spinorbit splittings having already been taken into account by the shifting operators, i.e., their influence on the relative energies of the spin-free states. A further manifestation of the correct performance of the shifting operators is the fact that the energies of the spin-orbit states show a lower quality as to the upper states, which is clear from Fig. 1, in accordance with the fact that the shifting terms have been truncated after ${ }^{5} \mathrm{D}$ at $2.5 \mathrm{eV}$. An addition of new terms should improve the results. We have included several, up to $b^{3} \mathrm{H}$ (see Table II), and we display the results in parentheses in Table III. This additionally enhances the energies below $2 \mathrm{eV}$ (especially improving the $J=0$ state) and significantly improves the results above $2 \mathrm{eV}$, showing the systematic character of this approach. In order to check the convergence of the energies with the number of terms included in the shifting operator and the completeness of the subspace defined by the spinfree CI wave functions we have analyzed the empirical-sfss $\mathrm{CI}(\mathrm{S})$ wave functions in terms of contributions from the spinfree CI wave functions and the residues. The result of this analysis is presented in Table IV. It is observed that large residues, i.e., large contributions from the space not spanned by the spin-free CI wave functions used in the shifting operator may be taken as an indication for the need of additional terms in the shifting operator. 


\section{CONCLUSIONS}

We have demonstrated that the one-electron WoodBoring spin-orbit operator used here is high of quality, even better than previously estimated from spin-orbit CI calculations which have been contaminated by an incomplete treatment of correlation. ${ }^{6,18}$ The use of an empirical scaling parameter thus appears to be superfluous to correct the spinorbit operator. Furthermore electron correlation effects as well as spin-orbit effects can be decoupled to a large extent by means of the spin-free-state-shifting technique. Spin-orbit CI calculations with a reference of the relevant configurations $\left[(5 d, 6 s)^{8}\right.$ in this case] plus single excitations produce very good spin-orbit splittings between the chemically relevant states of $\mathrm{Ir}^{+}$, provided that the spin-free effects, especially the correlation effects, are accurately taken into account. This implies that a convenient strategy for $a b$ initio spin-orbit molecular calculations involving $\mathrm{Ir}^{+}$is to pay special attention to the treatment of the correlation effects at the spin-free level, and to subsequently use a $s f s s$ technique in a multireference spin-orbit $\mathrm{CI}(\mathrm{S})$ calculation. In our opinion, these conclusions can be safely extended to the complete third series of transition metal elements. This strategy might be applied to many atoms and molecules; in such future cases the most demanding part of an ab initio calculation with spin-orbit effects would be the treatment of the correlation effects but these could be handled at a spin-free level. Limitations of the procedure might occur for cases for which a very large spin-orbit polarization exists. In these cases, a spin-free-state-shifted spin-orbit CASSCF procedure might be indicated.

\section{ACKNOWLEDGMENTS}

The authors would like to thank Dr. P. H. M. Uylings, from the University of Amsterdam, the Netherlands, for performing the spin-free empirical calculations and M. Gastreich (University of Bonn, Germany) for proofreading the manuscript. This work was partly supported by grants from the Spanish Ministerio de Educacion y Cultura (PB95-0201 and Acciones Integradas HA96-15), the Schwerpunktprogramm "Relativistische Effekte" of the Deutsche Forschungsgemeinschaft, DFG, (Ma 1051/2-2), the Deutscher Akademischer Austauschdienst, DAAD, (Acciones Integradas 314-AI-e-dr), and the European Science Foundation in the framework of the REHE program.

\footnotetext{
${ }^{1} \mathrm{~J}$. Almlöf and O. Gropen, in Reviews in Computational Chemistry, edited by K. B. Lipkowitz and D. B. Boyd (VCH, New York, 1996), Vol. 8, and references therein.

${ }^{2}$ L. Visscher, T. Saue, W. C. Nieuwpoort, K. Faegri, and O. Gropen, J. Chem. Phys. 99, 6704 (1993).
}

${ }^{3}$ C. Teichteil, M. Pelissier, and F. Spiegelmann, Chem. Phys. 81, 273 (1983).

${ }^{4}$ R. Pitzer and N. Winter, J. Phys. Chem. 92, 3061 (1988).

${ }^{5}$ A. B. Alekseyev, R. J. Buenker, H.-P. Liebermann, and G. Hirsch, J. Chem. Phys. 100, 2989 (1994).

${ }^{6}$ L. Seijo, J. Chem. Phys. 102, 8078 (1995).

${ }^{7}$ S. Katsuki and S. Huzinaga, Chem. Phys. Lett. 147, 597 (1988).

${ }^{8}$ S. Huzinaga, J. Mol. Struct.: THEOCHEM 80, 51 (1991).

${ }^{9}$ R. E. Moss, Advanced Molecular Quantum Mechanics, Studies in Chemical Physics (Chapman and Hall, London, 1973).

${ }^{10}$ J. H. Wood and A. M. Boring, Phys. Rev. B 18, 2701 (1978).

${ }^{11}$ A. M. Boring and J. H. Wood, J. Chem. Phys. 71, 32 (1979).

${ }^{12}$ J.-L. Heully, J. Phys. B: At. Mol. Phys. 15, 4079 (1982).

${ }^{13}$ J.-L. Heully and S. Salomonson, J. Phys. B: At. Mol. Phys. 15, 4093 (1982).

${ }^{14}$ M. Blume and R. E. Watson, Proc. R. Soc. London, Ser. A 270, 127 (1962).

${ }^{15}$ M. Blume and R. E. Watson, Proc. R. Soc. London, Ser. A 271, 565 (1963).

${ }^{16}$ W. Küchle, M. Dolg, H. Stoll, and H. Preuss, Mol. Phys. 74, 1245 (1991).

${ }^{17}$ M. Casarrubios and L. Seijo, J. Mol. Struct. THEOCHEM (to be published).

${ }^{18}$ M. Casarrubios and L. Seijo, Chem. Phys. Lett. 236, 510 (1995).

${ }^{19}$ M. Casarrubios and L. Seijo (in preparation).

${ }^{20}$ C. M. Marian, J. Chem. Phys. 94, 5574 (1991).

${ }^{21}$ C. M. Marian, Ber. Bunsenges. Phys. Chem. 99, 254 (1995).

${ }^{22}$ R. Llusar, M. Casarrubios, Z. Barandiarán, and L. Seijo, J. Chem. Phys. 105, 1 (1996).

${ }^{23}$ R. D. Cowan and D. C. Griffin, J. Opt. Soc. Am. 66, 1010 (1976).

${ }^{24}$ S. Huzinaga, L. Seijo, Z. Barandiarán, and M. Klobukowski, J. Chem. Phys. 86, 2132 (1987).

${ }^{25}$ F. Rakowitz and C. M. Marian, Chem. Phys. 225, 223 (1997)

${ }^{26}$ B.A. Hess, C. M. Marian, U. Wahlgren, and O. Gropen, Chem. Phys. Lett. 251, 365 (1996).

${ }^{27}$ T. van Kleef and B. Metsch, Physica B \& C 95, 251 (1978).

${ }^{28}$ J.-F. Wyart, A. Raassen, P. Uylings, and Y. Joshi, Phys. Scr. T47, 59 (1993).

${ }^{29} \mathrm{P}$. Uylings (private communication).

${ }^{30}$ Gaussian Basis Sets for Molecular Calculations, edited by S. Huzinaga (Elsevier, Amsterdam, 1984).

${ }^{31}$ MOLCAS version 3, K. Andersson, M. R. A. Blomberg, M. P. Fülscher, V. Kellö, R. Lindh, P.-Å. Malmqvist, J. Noga, J. Olsen, B. O. Roos, A. J. Sadlej, P. E. M. Siegbahn, M. Urban, P.-O. Widmark, University of Lund, Sweden, 1994.

${ }^{32}$ B. O. Roos, P. R. Taylor, and P. E. M. Siegbahn, Chem. Phys. 48, 157 (1980).

${ }^{33}$ P.E. M. Siegbahn, A. Heiberg, B. O. Roos, and B. Levy, Phys. Scr. 21, 323 (1980).

${ }^{34}$ P. E. M. Siegbahn, A. Heiberg, J. Almlöf, and B. O. Roos, J. Chem. Phys. 74, 2384 (1981).

${ }^{35}$ R. Ahlrichs, P. Scharf, and C. Ehrhardt, J. Chem. Phys. 82, 890 (1985).

${ }^{36}$ R. J. Gdanitz and R. Ahlrichs, Chem. Phys. Lett. 143, 413 (1988).

${ }^{37}$ COLUMBUS suite of programs (ARGOS, CNVRT, SCFPQ, LSTRN, CGDBG, and CIDBG). R. M. Pitzer (principal author). See: A. H. H. Chang and R. M. Pitzer, J. Am. Chem. Soc. 111, 2500 (1989), and references therein for a description. CNVRT and LSTRN have been adapted to handle ECPAIMP (Ref. 38) integrals by L. Seijo. CIDBG has been modified for spin-free-stateshifted spin-orbit CI calculations by M. Casarrubios.

${ }^{38}$ ECPAIMP is an integral program for ECP and AIMP calculations written by L. G. M. Pettersson, L. Seijo, and M. A. Nygren. 